\title{
Clinical Application of AIMS65 Scores to Predict Outcomes in Patients with Upper Gastrointestinal Hemorrhage
}

\author{
Ragesh Babu Thandassery, Manik Sharma, Anil K John, Khalid Mohsin Al-Ejji, Hamidulla Wani, Khaleel Sultan, \\ Muneera Al-Mohannadi, Rafie Yakoob, Moutaz Derbala, Nazeeh Al-Dweik, Muhammed Tariq Butt and Saad Rashid Al-Kaabi \\ Division of Gastroenterology, Department of Medicine, Hamad General Hospital, Doha, Qatar
}

Background/Aims: To evaluate the ability of the recently proposed albumin, international normalized ratio (INR), mental status, systolic blood pressure, age $>65$ years (AIMS65) score to predict mortality in patients with acute upper gastrointestinal bleeding (UGIB). Methods: AIMS65 scores were calculated in 251 consecutive patients presenting with acute UGIB by allotting 1 point each for albumin level $<30 \mathrm{~g} / \mathrm{L}, \mathrm{INR}>1.5$, alteration in mental status, systolic blood pressure $\leq 90 \mathrm{~mm} \mathrm{Hg}$, and age $\geq 65$ years. Risk stratification was done during the initial 12 hours of hospital admission.

Results: Intensive care unit (ICU) admission, endoscopic therapy, or surgery were required in 51 patients (20.3\%), 64 (25.5\%), and 12 $(4.8 \%)$, respectively. The predictive accuracy of AIMS65 scores $\geq 2$ was high for blood transfusion (area under the receiver operator characteristic curve [AUROC], 0.59), ICU admission (AUROC, 0.61), and mortality (AUROC, 0.74). The overall mortality was $10.3 \%$ $(n=26)$, and was $3 \%, 7.8 \%, 20 \%, 36 \%$, and $40 \%$ for AIMS65 scores of $0,1,2,3$, and 4 , respectively; these values were significantly higher in those with scores $\geq 2(30.9 \%)$ than in those with scores $<2(4.5 \%, p<0.001)$.

Conclusions: AIMS65 is a simple, accurate, non-endoscopic risk score that can be applied early (within 12 hours of hospital admission) in patients with acute UGIB. AIMS65 scores $\geq 2$ predict high in-hospital mortality. Clin Endosc 2015;48:380-384

Key Words: AIMS65 score; Upper gastrointestinal bleed; Endoscopy; Mortality

\section{INTRODUCTION}

Upper gastrointestinal bleeding (UGIB) is a gastrointestinal emergency that can result in significant morbidity, mortality, and use of health care resources. The etiology of UGIB can vary from trivial causes like gastric erosions to potentially fatal conditions like aorto-enteric fistula. UGIB therefore raises significant concern upon presentation in emergency departments. Accurate risk assessment for triaging and prognostication is extremely important. Accurate risk stratification will

Received: September 17, 2014 Revised: December 5, 2014 Accepted: December 26, 2014

Correspondence: Ragesh Babu Thandassery

Division of Gastroenterology, Department of Medicine, Hamad General Hospital, 2 South 2, Endoscopy unit, PO Box 3050, Doha, Qatar

Tel: +974-44392532, Fax: +974-44392279, E-mail: doc.ragesh@gmail.com

(cc This is an Open Access article distributed under the terms of the Creative Commons Attribution Non-Commercial License (http://creativecommons.org/ licenses/by-nc/3.0) which permits unrestricted non-commercial use, distribution, and reproduction in any medium, provided the original work is properly cited. enable urgent endoscopy and intensive care monitoring for high-risk patients, and facilitate discharge of low-risk patients from emergency care units. Various risk-scoring systems have been used to predict clinical outcomes in patients with UGIB. Most are cumbersome, require multiple variables including endoscopic appearance, and cannot be applied early. Rapid risk assessment in emergency settings is difficult in most of these scoring systems. The most widely applied scoring systems include the Glasgow-Blatchford Bleeding Score (GBS) ${ }^{2}$ and the clinical Rockall score. ${ }^{3}$ These scores utilize only pre-endoscopy criteria; however, the full Rockall score also utilizes endoscopic criteria. ${ }^{4}$ There are no clear data showing that these scoring systems successfully predict clinical outcomes. A recently proposed scoring system, AIMS65, was found to be a simple, accurate risk score to predict in-hospital mortality, length of hospital stay, and health care costs in patients with acute UGIB. The AIMS65 consists of the following components: albumin level $<3.0 \mathrm{~g} / \mathrm{dL}(\mathrm{A})$, international nor- 
malized ratio (INR) $>1.5$ (I), altered mental status (M), systolic blood pressure $\leq 90 \mathrm{~mm} \mathrm{Hg}(\mathrm{S})$, and age $>65$ years (65). When more than two components of the AIMS65 are present, the mortality risk is considered to be high. ${ }^{5}$ Another study from Saltzman et al. ${ }^{5}$ showed the AIMS65 score to be superior to the GBS in predicting inpatient mortality from UGIB, whereas the GBS was superior for predicting the need for blood transfusion. ${ }^{6}$ In the current study, we applied the AIMS65 to patients with UGIB to evaluate its predictive accuracy for blood transfusion, endoscopic therapy, intensive care unit (ICU) admission, and surgery, as well as predicting rebleeding, length of hospital stay, and mortality.

\section{MATERIALS AND METHODS}

We retrospectively evaluated patients presenting with UGIB who underwent endoscopy from January to December 2012. This study was performed in a tertiary care academic center in Doha, Qatar with approval from the Institute Research Committee (IRB number, RP: 14186/14). The inclusion criteria were: (1) all patients above 14 years of age, (2) acute UGIB presenting within 24 hours of hemorrhage onset, and (3) endoscopic evaluation within 12 hours of hospital admission. The exclusion criterion was patients with late presentation $(>24$ hours) after UGIB onset.

UGIB was diagnosed based on clinical presentation of coffee ground vomiting, hematemesis, melena, and the presence of blood in nasogastric aspirate. All patients with UGIB are admitted to the emergency unit of our hospital and undergo routine laboratory evaluation with complete blood count, coagulation profile, and basic biochemistry. Gastroenterologists on call are informed immediately, and patients with significant hemorrhage undergo urgent endoscopic evaluation within 12 hours. Patients receive resuscitative measures and are transferred to the ICU when they have signs of shock or altered sensorium. Patients with massive hemorrhage with risk of aspiration undergo prophylactic endotracheal intubation. We retrospectively recorded and analyzed baseline clinical data, laboratory reports, transfused blood units, endoscopic records, and subsequent follow-ups until patient death or discharge.

All patients with non-variceal UGIB in our emergency department were started on oral proton pump inhibitors before undergoing endoscopy, followed by doses at 6-hour intervals (every 6 hours). The duration of treatment was dependent on the endoscopic findings and clinical course. Intravenous terlipressin (2 mg every 6 hours) was started in all patients with suspected variceal bleeding. Blood transfusion was indicated for UGIB patients with hemoglobin less than $8 \mathrm{~g} / \mathrm{dL}$ or with signs of hemodynamic instability despite fluid resuscitation. The decision to transfuse blood was made on a case-to-case basis depending on the patient's cardiac status and the presence of comorbidities. AIMS65 scores were calculated by allotting 1 point each for albumin (A) levels $<30 \mathrm{~g} / \mathrm{L}$, INR (I) > 1.5 , alteration in mental status (M), systolic blood pressure (S) $\leq 90 \mathrm{~mm} \mathrm{Hg}$, and age $\geq 65$ years. The predictive accuracy of the AIMS65 score for mortality was calculated. Other outcome measures indicating the course of illness were also calculated, including blood transfusion, endoscopic therapy, ICU admission, and surgery, as well as the incidence of rebleeding and duration of hospital and ICU stay.

\section{Data analysis}

Statistical analysis was performed using IBM SPSS version 22.0 (IBM Co., Armonk, NY, USA). Data were evaluated for any outliers, errors, and missing values. When the data were normally distributed, continuous variables were compared using the Student $t$-test. For more than two groups, one-way

Table 1. Characteristics of Patients with Upper Gastrointestinal Bleeding $(n=251)$

\begin{tabular}{lc}
\hline Characteristic & Value \\
\hline Age, yr & $52(15-84)$ \\
\hline Male to female ratio & $3.3: 1$ \\
\hline Etiology of hemorrhage & \\
\hline Gastric ulcer & $34(13.5)$ \\
\hline Duodenal ulcer & $70(27.8)$ \\
\hline Both gastric and duodenal ulcers & $4(1.6)$ \\
\hline Gastroduodenal erosions & $27(10.7)$ \\
\hline Esophageal varices & $32(12.7)$ \\
\hline Miscellaneous & $36(14.3)$ \\
\hline Endoscopic therapy & $64(25.5)$ \\
\hline Endoscopic variceal ligation & $19(7.5)$ \\
\hline Combined (heater probe and Injection & $19(7.5)$ \\
\hline sclerotherapy) & $13(5.1)$ \\
\hline Injection sclerotherapy & $7(2.7)$ \\
\hline Hemoclips & $12(4.7)$ \\
\hline Surgery & $184(73.3)$ \\
\hline Blood transfusion requirement & $21(8.3)$ \\
\hline Rebleeding & $54(21.5)$ \\
\hline Mntensive care unit admission & $4.6 \pm 5.4$ \\
\hline Mean intensive care unit stay, day & $10.6 \pm 16.9$ \\
\hline Mortality & $26(10.3)$ \\
\hline Vital stay, day & \\
\hline
\end{tabular}

Values are presented as median (range), number of patients (\%), or mean \pm SD. 
analysis of variance was used. The Mann-Whitney test was used for analysis of skewed (non-normal) data. Quantitative data was described as mean and standard deviation with 95\% confidence intervals (CIs). Categorical data was shown as proportions. Comparison of various categorical variables between surviving and deceased patient was performed using chi-square test of association to identify statistical associations. Spearman correlation coefficients were also calculated between different quantitative variables. Receiver operating characteristic curves were calculated to assess the predictive accuracy of the AIMS65 score for mortality.

\section{RESULTS}

This study included 251 patients who met the inclusion and exclusion criteria. Their median age was 52 years (range, 15 to 84), including 193 men. The etiology for UGIB was duodenal ulcer, gastric ulcer, and esophageal varices in 70 patients (27.8\%), 34 (13.5\%), and 32 (12.7\%), respectively (Table 1). Blood transfusion was required in 184 patients (73.3\%; median 2 units of packed red blood cells; range, 1 to 45). Among patients who underwent endoscopy, endoscopic therapy was required in 64 patients $(25.5 \%)$. ICU admission was required for 54 patients (21.5\%), while 21 (8.3\%) had rebleeding and 12 (4.7\%) underwent surgery for refractory bleeding. The mean hospital and ICU stays were $10.6 \pm 16.9$ and $4.6 \pm 5.4$ days, respectively. The overall mortality was $10.3 \%(n=26)$.

In patient groups with AIMS65 scores $<2$ and $\geq 2$, there were no significant differences between the need for endoscopic therapy ( $26.1 \%$ vs. $21.8 \%, p=0.42)$, surgery ( $6.1 \%$ vs. $0 \%$, $p=0.06)$, and rebleeding ( $7.7 \%$ vs. $10.1 \%, p=0.44)$. However, blood transfusion ( $69.4 \%$ vs. $87.3 \%, p=0.008)$, ICU stay $(16.8 \%$ vs. $38.2 \%, p=0.001)$, and mortality were significantly higher in the latter group. The mortality among patients with AIMS65 scores of $0,1,2,3$, and 4 were $3 \%, 7.8 \%, 20 \%, 36 \%$, and $40 \%$, respectively (Table 2). The mortality was significantly higher in those with scores $\geq 2$ (30.9\%) compared to those with scores $<2(4.5 \%, p<0.001)$. The predictive accuracy for mortality with scores $\geq 2$ was high (area under the receiver operator characteristic curve [AUROC], 0.74; 95\% CI, 0.63 to 0.85 ) (Table 3). Similarly, the AUROC for AIMS65 scores for predicting blood transfusion $(0.60 ; 95 \%$ CI, 0.51 to 0.67$)$ and ICU stay $(0.61$; $95 \%$ CI, 0.52 to 0.70 ) were also high. The mean hospital stay (21.5 \pm 31.1 days vs. $9.0 \pm 12.8$ days, $p=0.04)$ and ICU stay $(5.1 \pm 6.1$ days vs. $3.5 \pm 3.6$ days, $p=0.042$ ) were significantly higher in patients with scores $\geq 2$ compared to those with scores $<2$.

\section{DISCUSSION}

International consensus statements as well as American

Table 2. Required Interventions, Blood Transfusions, and Mortality among Groups with Different AIMS65 Scores

\begin{tabular}{|c|c|c|c|c|c|c|}
\hline \multirow{2}{*}{ Variable } & \multicolumn{5}{|c|}{ AIMS65 score } & \multirow{2}{*}{$p$-value } \\
\hline & $\mathbf{0}$ & 1 & 2 & 3 & 4 & \\
\hline Blood transfusion & $90(68.2)$ & $46(71.9)$ & $17(85)$ & $22(88)$ & $9(90)$ & 0.11 \\
\hline Endoscopic intervention & $37(28)$ & $15(23.4)$ & $5(25)$ & $5(20)$ & $2(20)$ & 0.88 \\
\hline ICU admission & $11(8.3)$ & $22(34.4)$ & $5(25)$ & $12(48)$ & $4(40)$ & $<0.001$ \\
\hline Rebleeding & $8(6.1)$ & $7(10.9)$ & $3(15)$ & $1(4)$ & $2(20)$ & 0.29 \\
\hline Surgery & $6(4.5)$ & $6(9.4)$ & 0 & 0 & 0 & 0.22 \\
\hline Mortality & $4(3)$ & $5(7.8)$ & $4(20)$ & $9(36)$ & $4(40)$ & $<0.001$ \\
\hline
\end{tabular}

Values are presented as number (\%).

ICU, intensive care unit.

Table 3. Predictive Accuracy of AIMS65 Scores $\geq 2$ for Different Outcome Measures

\begin{tabular}{lccc}
\hline Outcome measures & AUROC $(\mathbf{9 5 \%}$ CI) & Standard error & $p$-value \\
\hline Blood transfusion & $0.60(0.51-0.67)$ & 0.041 & 0.04 \\
Endoscopic intervention & $0.48(0.39-0.56)$ & 0.043 & 0.59 \\
ICU admission & $0.61(0.52-0.70)$ & 0.046 & 0.01 \\
Rebleeding & $0.53(0.40-0.66)$ & 0.068 & 0.06 \\
Surgery & $0.38(0.25-0.52)$ & 0.170 & 0.18 \\
Mortality & $0.74(0.63-0.85)$ & 0.057 & $<0.001$ \\
\hline
\end{tabular}

AUROC, area under the receiver operator characteristic curve; $\mathrm{CI}$, confidence interval; ICU, intensive care unit. 
College of Gastroenterology practice guidelines have emphasized the application of prognostic scores for the management of UGIB. ${ }^{7-9}$ A score predictive of different outcome measures is extremely important in prognostication and making important therapeutic decisions. Most previous studies have assessed GBS and Rockall scores. ${ }^{2,310-12}$ The GBS was shown to be similar or even superior to the Rockall score in this context., ${ }^{2,13}$ However, the GBS is difficult to apply in routine clinical practice, as it is weighted and incorporates diverse variables like patient medical history and laboratory data, some of which are not clearly defined. The AIMS65 score was proposed as a simple non-invasive pre-endoscopic score to predict in-hospital mortality, length of hospital stay, and healthcare cost in patients with acute UGIB. ${ }^{5}$ Several studies have evaluated the predictive accuracy of this new score for different outcome measures, with variable results. ${ }^{6,14-17}$

Our study of 251 patients is the first report on prognostication of UGIB from the Middle East; we found that the AIMS65 is a simple, non-endoscopic risk score that can be applied early (within 12 hours of hospital admission) in patients with acute UGIB. In this study, the score predicted high in-hospital mortality, blood transfusion, ICU admission, and duration of hospital and ICU stays. However, the need for endoscopic intervention and surgery and the incidence of rebleeding were comparable between groups with AIMS65 scores $<2$ and $\geq 2$, which could be due to the relatively lower number of patients in these subgroups. The predictive accuracy of the AIMS65 score for mortality in our study was high and comparable to that of the validation cohort in the study by Saltzman et al. ${ }^{5}$ Saltzman et al. ${ }^{5}$ reported that the patients in the validation cohort with no risk factors had a low mortality rate $(0.3 \%)$ and those with all five risk factors had a high mortality rate (24.5\%). Higher numbers of risk factors were associated with longer length of stay and higher costs in both the derivation and validation cohorts. ${ }^{5}$ Our results generally agreed with these findings. Our study group had lower proportion of patients with non-variceal bleeding (87.2\%) compared to the study by Saltzman et al. ${ }^{5}(98.3 \%)$. In a study by Jung et al. ${ }^{14}$ that included only patients with bleeding peptic ulcers; however, the AIMS65 score showed lower predictive accuracy for clinical outcomes. Their single center retrospective study ( $n=149$ ) of patients with bleeding peptic ulcers found that the AIMS65 score was not suitable for predicting outcomes in this patient population (area under the curve, $0.571 ; 95 \%$ CI, 0.49 to 0.65 ). They found low serum albumin levels to be a risk factor associated with high mortality in these patients. They explained the discordance in their results by the diverse etiology (mixed patient population with both variceal and non-variceal UGIB) for GI hemorrhage in the study by Saltzman et al. ${ }^{5}$ In a comparative study of AIMS65 score and GBS, Hyett et al. ${ }^{6}(n=278)$ found that the AIMS65 score was superior in predicting inpatient mortality from UGIB, while the GBS was superior for predicting the need for blood transfusion. Both scores were similar in predicting the composite clinical endpoint (inpatient mortality; rebleeding; and endoscopic, radiologic, or surgical intervention), ICU admission, rebleeding, length of stay, and timing of endoscopy. Chandra ${ }^{15}$ reported that the AIMS65 score accurately predicted 30- and 90-day all-cause mortality among patients with UGIB.

In another retrospective study, Nakamura et al. ${ }^{16}$ evaluated the role of AIMS65 in both upper $(n=130)$ and lower $(n=102)$ GI hemorrhage. The AIMS65 and GBS scores were higher in the upper GI hemorrhage group compared to the lower GI hemorrhage group. They showed that blood transfusion, co-existing malignancy, absence of endoscopic hemostasis, and high AIMS65 score were independent prognostic factors for poor overall survival. They also found that the AIMS65 score, but not GBS, was an independent prognostic factor for overall survival. They reported AIMS65 to be the best score for predicting outcomes with acute GI bleeding. However, they could not explain the reason for the differences between the two scoring systems. They speculated that all of the components included in the AIMS65 seemed more appropriate as prognostic scores as compared to the GBS. They also found that older age ( $>65$ years) or low albumin levels $(<3.0 \mathrm{~g} / \mathrm{dL})$ might have directly affected patient outcomes. Masaoka and Suzuki ${ }^{17}$ recently proposed a combined algorithm incorporating both AIMS65 and GBS score to assess patients with suspected UGIB in emergency room settings. In this algorithm, an initial GBS screen is performed; those with low risk (GBS $\leq 2$ ) receive conservative medical treatment, while high-risk patients (GBS $>2$ ) receive further assessment of mortality risk based on AIMS65 score. However, this dual scoring approach requires validation. Except for the study by Jung et al., ${ }^{14}$ all other studies including ours showed that AIMS65 has high predictive accuracy for mortality.

Early risk stratification may not reflect the final outcome as this time point may be too soon for comprehensive evaluation. In order to identify the most appropriate "early" time point for applying this score, the AIMS65 needs to be serially applied to plot the course of illness. Because our study included only patients that underwent endoscopy, we could not apply the score to predict the need for endoscopy in patients presenting with UGIB. Our study was retrospective with a limited sample size. Larger multicenter prospective studies that assess multiple outcome measures with head-to-head comparison of other clinical scores will help us to further establish the exact role of this new scoring system. Future studies should also evaluate the role of combining multiple prognostic scores to improve 
the predictive accuracy.

In conclusion, the AIMS65 is a simple, accurate, non-endoscopic risk score that can be applied to patients with acute UGIB. This score can be used successfully within as little as 12 hours after hospital admission, thus assisting in early decision-making and triage. AIMS65 scores $\geq 2$ predict high in-hospital mortality, blood transfusion, ICU admission, and increased duration of hospital and ICU stays.

\section{Conflicts of Interest}

The authors have no financial conflicts of interest.

\section{Acknowledgments}

This abstract in a preliminary form was presented at Digestive Disease Week (DDW) 2014, and accepted as oral presentation at United European Gastroenterology Week (UEGW) 2014 (Vienna).

\section{REFERENCES}

1. Loperfido S, Baldo V, Piovesana E, et al. Changing trends in acute upper-GI bleeding: a population-based study. Gastrointest Endosc 2009;70:212-224

2. Stanley AJ, Ashley D, Dalton HR, et al. Outpatient management of patients with low-risk upper-gastrointestinal haemorrhage: multicentre validation and prospective evaluation. Lancet 2009;373:42-47.

3. Rockall TA, Logan RF, Devlin HB, Northfield TC. Risk assessment after acute upper gastrointestinal haemorrhage. Gut 1996;38:316-321.

4. Vreeburg EM, Terwee CB, Snel P, et al. Validation of the Rockall risk scoring system in upper gastrointestinal bleeding. Gut 1999;44:331-335.

5. Saltzman JR, Tabak YP, Hyett BH, Sun X, Travis AC, Johannes RS. A simple risk score accurately predicts in-hospital mortality, length of stay, and cost in acute upper GI bleeding. Gastrointest Endosc 2011;74:12151224.
6. Hyett BH, Abougergi MS, Charpentier JP, et al. The AIMS65 score compared with the Glasgow-Blatchford score in predicting outcomes in upper GI bleeding. Gastrointest Endosc 2013;77:551-557.

7. Adams BD, McHugh KJ, Bryson SA, Dabulewicz J. The law of unintended consequences: the Joint Commission regulations and the digital rectal examination. Ann Emerg Med 2008;51:197-201, 201 e191.

8. Le Jeune IR, Gordon AL, Farrugia D, Manwani R, Guha IN, James MW. Safe discharge of patients with low-risk upper gastrointestinal bleeding (UGIB): can the use of Glasgow-Blatchford Bleeding Score be extended? Acute Med 2011;10:176-181.

9. Stephens JR, Hare NC, Warshow U, et al. Management of minor upper gastrointestinal haemorrhage in the community using the Glasgow Blatchford Score. Eur J Gastroenterol Hepatol 2009;21:1340-1346.

10. Dicu D, Pop F, Ionescu D, Dicu T. Comparison of risk scoring systems in predicting clinical outcome at upper gastrointestinal bleeding patients in an emergency unit. Am J Emerg Med 2013;31:94-99.

11. Wang CH, Chen YW, Young YR, Yang CJ, Chen IC. A prospective comparison of 3 scoring systems in upper gastrointestinal bleeding. Am J Emerg Med 2013;31:775-778.

12. Bryant RV, Kuo P, Williamson K, et al. Performance of the GlasgowBlatchford score in predicting clinical outcomes and intervention in hospitalized patients with upper GI bleeding. Gastrointest Endosc 2013;78:576-583.

13. Chen IC, Hung MS, Chiu TF, Chen JC, Hsiao CT. Risk scoring systems to predict need for clinical intervention for patients with nonvariceal upper gastrointestinal tract bleeding. Am J Emerg Med 2007;25:774-779.

14. Jung $\mathrm{SH}, \mathrm{Oh} \mathrm{JH}$, Lee HY, et al. Is the AIMS65 score useful in predicting outcomes in peptic ulcer bleeding? World J Gastroenterol 2014;20:18461851.

15. Chandra S. AIMS65 score predicts short-term mortality but not the need for intervention in acute upper GI bleeding. Gastrointest Endosc 2013;78:381-382.

16. Nakamura S, Matsumoto T, Sugimori H, Esaki M, Kitazono T, Hashizume M. Emergency endoscopy for acute gastrointestinal bleeding: prognostic value of endoscopic hemostasis and the AIMS65 score in Japanese patients. Dig Endosc 2014;26:369-376.

17. Masaoka T, Suzuki H. Does the AIMS65, a new risk score for upper gastrointestinal bleeding, work in Japan? Dig Endosc 2014;26:331-332. 